\section{Osteocondrodisplasia em um felino jovem da raça Scottish Fold: relato de caso}

\author{
Osteochondrodysplasia in a young cat of the Scottish Fold \\ breed: case report
}

Mariana Palha de Brito Jardim*, Luiza Freire de Farias', Gabriela de Carvalho Cid², Heloisa Justen Moreira de Souza ${ }^{3}$, Katia Barão Corgozinho ${ }^{4} \&$ Ricardo Siqueira da Silva ${ }^{5}$

'Médica veterinária, Mestranda em Patologia e Ciências Clínicas. Programa de Pós-graduação em Medicina Veterinária, Universidade Federal Rural do Rio de Janeiro - UFRRJ, Seropédica, RJ, Brasil

2Médica veterinária, Doutoranda em Patologia e Ciências Clínicas. Programa de Pós-graduação em Medicina Veterinária, Universidade Federal Rural do Rio de Janeiro - UFRRJ, Seropédica, RJ, Brasil

${ }^{3}$ Médica veterinária, Professora associada da disciplina Patologia Clínica e Cirúrgica. Universidade Federal Rural do Rio de Janeiro - UFRRJ, Seropédica, RJ, Brasil

${ }^{4}$ Médica veterinária, Doutora em Clínica e Reprodução Animal. Universidade Federal Fluminense - UFF, Niterói, RJ, Brasil

${ }^{5}$ Médico veterinário, Professor associado da disciplina Patologia Clínica e Cirúrgica. Universidade Federal Rural do Rio de Janeiro - UFRRJ, Seropédica, RJ, Brasil

\section{Resumo}

Os gatos Scottish Fold possuem um gene autossômico dominante que determina a ocorrência das orelhas dobradas para frente e graus variados de osteocondrodisplasia. Objetiva-se com este trabalho relatar um caso de osteocondrodisplasia em um gato jovem da raça Scottish Fold, destacando as características clínicas, patológicas e a conduta terapêutica adotada mediante o quadro clínico, visando difundir informações relacionadas ás particularidades deste padrão racial felino. Foi levado para atendimento clínico um gato doméstico da raça Scottish Fold, macho, com 12 meses deidade, devido à queixa de dificuldade locomotora. Na avaliação radiográfica as articulações társicas apresentaram-se hipoplásicas, com angulação anormal do eixo nos metatarsos, falanges e articulações relacionadas, compatível com osteocondrodisplasia. Já nas articulações coxofemorais evidenciou-se displasia coxofemoral leve. O tratamento consistiu na utilização de gabapentina, sulfato de condroitina eômega-3. Esteé o primeiro relato de osteocondrodisplasia em um Scottish Fold no Brasil. As deformidades osteoarticulares observadas no felino jovem da raça Scottish Fold com osteocondrodisplasia constituem uma preocupação, em função do comprometimento da qualidade de vida do paciente e ausência de um tratamento curativo para tal afecção, assim o estudo pôde expandir informações a respeito das características inerentes a este padrão racial.

Palavras-chave: gatos, mutações genéticas, doenças osteoarticulares.

\begin{abstract}
Scottish Fold cats have an autosomal dominant gene that determines the occurrence of forward-bent ears and varying degrees of osteochondrodysplasia. The objective of this work is to report a case of osteochondrodysplasia in a young Scottish Fold cat, highlighting the clinical and pathological characteristics and the therapeutic behavior adopted by the clinical picture, aiming to disseminate information related to the particularities of this feline racial pattern. A Scottish Fold male domestic cat, 12 months old, was brought to clinical care due to the complaint of locomotor difficulty. In the radiographic evaluation, the tarsal joints were hypoplastic, with abnormal axis angulation in the metatarsals, phalanges and related joints, compatible with osteochondrodysplasia. Already in the hip joints, mild hip dysplasia was evident. Treatment consisted of gabapentin, chondroitin sulfate and omega-3. This is the first report of osteochondrodysplasia in a Scottish Fold in Brazil. The osteoarticular deformities observed in the Scottish Fold feline with osteochondrodysplasia are a concern, due to the patient's quality of life impairment and the absence of a curative treatment for this condition, so the study was able to expand information regarding the inherent characteristics of this disease racial pattern.
\end{abstract}

Keywords: cats, genetic mutations, osteoarticular diseases.

\section{BJ $\mathrm{M}$}

Brazilian Journal of Veterinary Medicine

p-ISSN 0100-2430

e-ISSN 2527-2179

ఠ
Como citar: Jardim, M. P. B., Farias, L. F., Cid, G. C., Souza, H. J. M., Corgozinho, K. B., \& Silva, R. S. (2017). Osteocondrodisplasia em um felino jovem da raça Scottish Fold: relato de caso. Brazilian Journal of Veterinary Medicine, 39(2), 133-137. doi: 10.29374/2527-2179.bjvm012217

Fonte de financiamento: Nenhuma.

Conflito de interesses: Os autores declaram não haver conflito de interesses que precisam ser informados.

Recebido: Dezembro 18, 2016.

Aceito: Março 23, 2017.

Oestudo foi realizado noSetor de Clínica Médica dos Gatos Domésticos, Hospital Veterinário de Pequenos Animais, Universidade Federal Rural do Rio de Janeiro - UFRRJ, Seropédica, RJ, Brasil.

\section{*Correspondência}

Mariana Palha de Brito Jardim Setor de Clínica Médica dos Gatos Domésticos, Instituto de Veterinária, Universidade Federal Rural do Rio de Janeiro - UFRRJ

Rodovia BR 465, Km 07, s/n, Zona Rural CEP 23899-000 - Seropédica (RJ), Brasil E-mail: jardim.marii@gmail.com
Copyright Jardim et al. Esteé um artigo publicado em acesso aberto (Open Access) sob a licença Creative Commons Attribution Non-Commercial, que permite uso, distribuição e reprodução em qualquer meio, sem restrições desde que sem fins comerciais e que o trabalho original seja corretamente citado. 


\section{Introdução}

Os felinos Scottish Folds possuem um gene autossômico dominante predominantemente incompleto, determinado atualmente como TRPV4. Esta mutação genética faz com que a cartilagem auricular se volte em direção aos olhos e rege a ocorrência de distúrbios de ossificação endocondral que ocorrem durante o crescimento destes animais, resultando em manifestações de deformidade osteoarticular como a osteocondrodisplasia (Partington et al., 1996; Malik et al., 1999; Gandolfi et al., 2016). Os gatos afetados possuem caudas curtas, grossas, inflexíveis e pés encurtados (Graeme, 2000).

Scottish Folds quando homozigotos tendem a expressar a osteocondrodisplasia de maneira mais grave, enquanto os heterozigotos também desenvolvem a afecção, porém de forma menos acentuada (Takanosu et al., 2008).

A raça foi banida na Inglaterra, porém se perpetuou nos Estados Unidos (Malik et al., 1999). Os gatos Scottish Folds despertam interesse entre tutores e comercializadores devido as suas características singulares que incluem orelhas dobradas, olhos redondos grandes, curva suave do nariz e membros grossos e curtos. Devido a sua aparência doce e personalidade amigável a raça é popular em diversos países (Malik, 2001; Gunn-Moore et al., 2008), tornando-se recentemente atrativa no Brasil. Destarte faz-se necessário a publicação de informações e estudos a cerca das anormalidades osteoarticulares desta raça de gatos.

Objetiva-se com este trabalho relatar um caso de osteocondrodisplasia em um gato jovem da raça Scottish Fold, destacando as características clínicas, patológicas e a conduta terapêutica adotada mediante o quadro clínico, visando difundir informações relacionadas ás particularidades deste padrão racial felino.

\section{Histórico}

Um gato doméstico, macho castrado, da raça Scottish Fold, com 12 meses de idade, com peso igual a 3,05 kg foi levado para atendimento clínico devido à queixa de claudicação.

O felino foi adquirido de um gatil comercializador da referida raça, situado em Portugal. Notou-se andar claudicante, encurtamento e deformação anatômica dos dígitos tarsais durante a deambulação do animal no consultório. Na palpação se verificou discreta sensibilidade dolorosa da porção distal de ambos membros pélvicos.

O paciente alimentava-se com ração comercialmente balanceada e própria para a sua faixa etária, á inspeção foi observado bom estado corpóreo, sem aparentes sinais de desnutrição. O gato se encontrava normocorado, normoidratado e sem alterações em demais parâmetros.

Na avaliação radiográfica as articulações társicas apresentaram-se hipoplásicas, com angulação anormal do eixo no segundo, quarto e quinto metatarsos esquerdos e no segundo e quinto metatarsos direitos, assim como em suas referentes falanges, com consequente angulação anormal das respectivas articulações metatarso-falangeanas, compatível com osteocondrodisplasia. Já nas articulações coxofemorais evidenciou-se incongruência entre as cabeças femorais e os acetábulos, representada por leve aumento do espaço articular (ângulo de Norberg aproximado de 100), compatível com displasia coxofemoral leve (Figura 1).

Na ultrassonografia abdominal, assim como no hemograma e bioquímicas séricas não foram relatadas alterações dignas de nota. O felino foi considerado negativo mediante o teste sorológico para o antígeno da leucemia e anticorpo da imunodeficiência felina.

O tratamento instituído preconizou a analgesia do paciente, assim, foi prescrito gabapetina (Barraderm Farmácia de Manipulação. Rio de Janeiro, RJ, Brasil), na dose de $5 \mathrm{mg} / \mathrm{kg}$, por via oral, a cada 24 horas por uso contínuo; quanto a suplementação nutricional, com intuito de auxiliar a regeneração das cartilagens, recomendou-se a utilização de sulfato de condroitina (CondroPlex 500 mg - Avert Laboratórios. Bragança Paulista, SP, Brasi Avert Laboratórios. Bragança Paulista, SP, Brasil), na dose de $500 \mathrm{mg} / g a t o$, a ser administrado por via oral, a cada 48 horas por uso contínuo e ômega-3 constituído por ácido eicosapentaenoico (EPA) e ácido docosahexaenóico (DHA) (Ograx-3500 mg ${ }^{\circledR}$ - Avert Laboratórios. Bragança Paulista, SP, Brasil Avert Laboratórios. Bragança Paulista, SP, Brasil), na dose de 500mg/gato, a ser administrado por via oral, a cada 24 horas, por uso contínuo. Até o momento da descrição deste relato não foram consideradas abordagens terapêuticas e/ou cirúrgicas mais invasivas devido ao grau de osteocondrodisplasia do animal. 


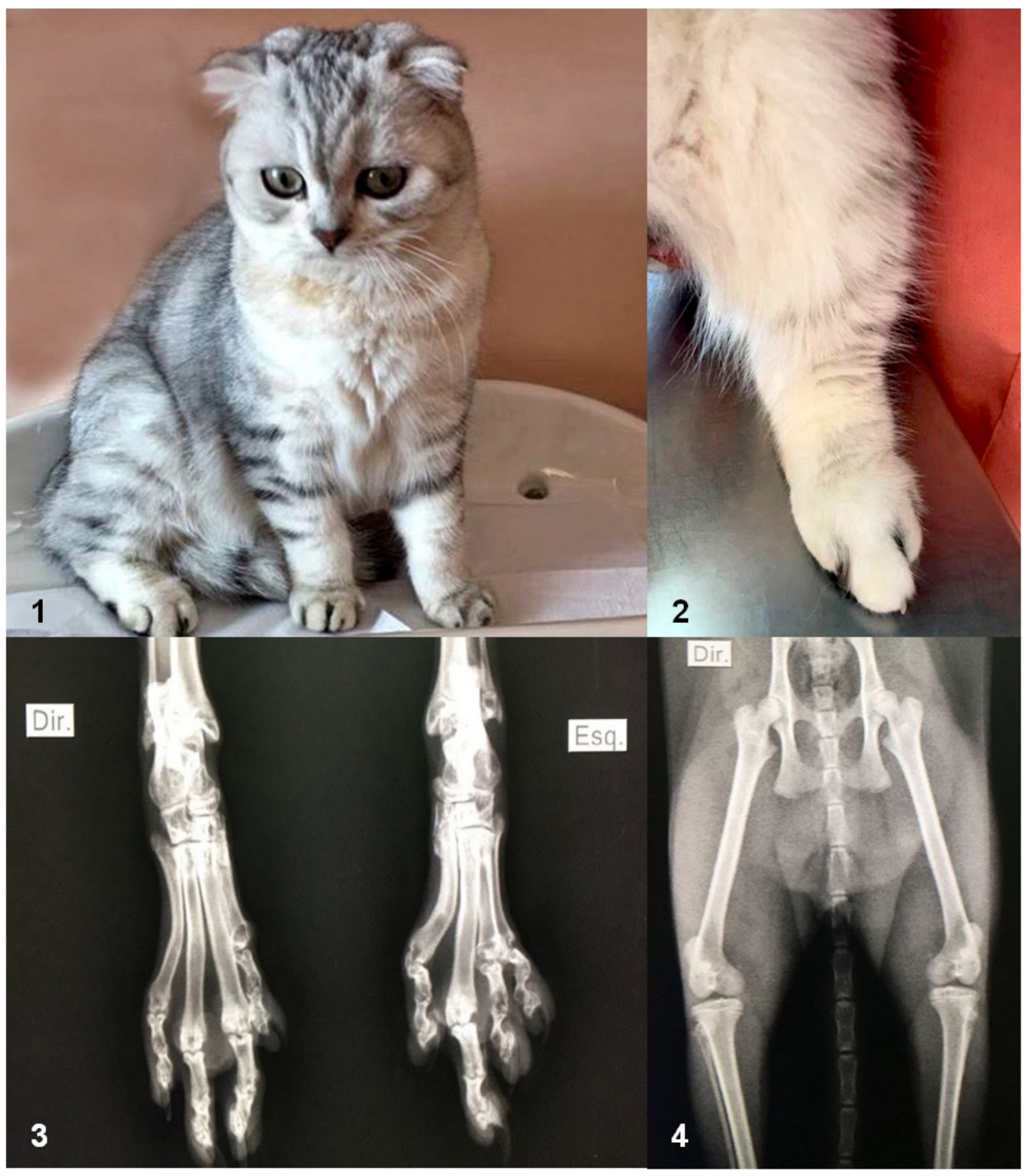

Figura 1. Osteocondrodisplasia em gato da raça Scottish Fold com 12 meses de idade. (1) Felino doméstico durante o exame físico com características compatíveis ao padrão racial; (2) Membro pélvico esquerdo com deformidade falangeana; (3) Hipoplasia com angulação do eixo ósseo no segundo, quarto e quinto metatarsos esquerdos e no segundo e quinto metatarsos direitos, assim como em suas referentes falanges, com consequente angulação anormal das respectivas articulações metatarso-falangeanas, compatível com osteocondrodisplasia; (4) Incongruência entre as cabeças femorais e os acetábulos, representada por leve aumento do espaço articular, compatível com displasia coxofemoral leve (ângulo de Norberg aproximado de 1000).

\section{Discussão}

As osteocondrodisplasias constituem um grupo heterogêneo de distúrbios causados por defeitos estruturais, metabólicos e endócrinos que comprometem a cartilagem e / ou o crescimento ósseo e dão origem a um esqueleto mal formado. O desenvolvimento esquelético éum processo altamente complexo influenciado por uma grande quantidade de genes e o ajuste preciso desses genes é essencial para o desenvolvimento normal do esqueleto (Gandolfi et al., 2016). No Scottish Fold a osteocondrodisplasia ocorre devido a alteração congênita degenerativa, resultando em defeitos morfológicos nos esqueletos axiais e apendiculares (Chang et al., 2007; Lascelles, 2010) a exemplo do caso relatado.

As deformidades progressivas na cauda e extremidades distais emergem quando os gatos têm cerca de sete semanas de idade e estas permanecem ao longo de suas vidas (Aydin et al., 2015). 
Como observado no presente estudo tais anomalias prejudicam a qualidade de vida de animais muito jovens e frustram consequentemente seus respectivos tutores.

Os felinos afetados pela afecção demonstram sinais de dor, claudicação, relutância em saltar e correr e andar rígido devido à propensão da raça quanto ao desenvolvimento de desordens musculoesqueléticas. A osteoartrite progressiva pode prejudicar até mesmo a socialização destes animais que tendem a se manter deitados e são caracterizados como animais de baixa vivacidade (Graeme, 2000; Chang et al., 2007; Takeuchi \& Mori, 2009; Inoue et al., 2016).

As características radiográficas incluem irregularidades no tamanho e na forma dos ossos carpais, tarsais, metacarpianos, metatarsianos, nas falanges e vértebras caudais, além de demonstrarem espaços articulares estreitos e formação progressiva de ossos novos em torno das articulações dos membros distais com osteopenia difusa do osso adjacente e formação de exostose plantar caudal ao calcâneo em casos avançados. De forma concordante ao caso relatado as deformidades radiográficas geralmente são mais encontradas nos membros pélvicos, como descrito por Graeme (2000).

O tratamento preconizado para a osteocondrodisplasia baseia-se principalmente no controle da dor e na regeneração das articulações, visando permitir o alívio da claudicação e relutância a movimentos, já que não há cura ou tratamento específico para a doença (Hubler et al., 2004; Chang et al., 2007).

A gabapetina, um fármaco da classe dos anticonvulsivantes, utilizada no controle da dor neuropática é recomendada para tratar da osteoartrite em gatos, devido à dor crônica que estes pacientes apresentam (Bennett et al., 2012). Tanto a condroitina quanto a glucosamina são empregadas no tratamento das artrites articulares por estarem envolvidas no metabolismo da matriz cartilaginosa, se estima assim o potencial de reparação da cartilagem e diminuição da sua degradação, sugere-se ainda o poder anti-inflamatório destas substâncias. Já os ácidos graxos omêga 3 visam melhorar a atividade dos felinos com artrite degenerativa (Davies, 2016; Bennett et al., 2012; Aydin et al., 2015).

Ressalta-se ainda a adaptação do ambiente a condição de animais com osteoartrite e a adequação de determinados hábitos, incluindo escovações diárias, colocação de suportes de elevação nos comedouros e bebedouros, aquisição de vasilhas de eliminação mais rasas, utilização de degraus e/ou rampas de acesso aos locais elevados nos quais o animal costuma pular para acessar, emprego de materiais acolchoados sobre os locais de repouso, arranhadores horizontais, bem como prevenção e tratamento da obesidade e controle de exercícios moderados (Hardie, 1997; Bennett et al., 2012; Davies, 2016; Frye et al., 2016).

Em casos onde as manifestações clínicas da osteocondrodisplasia demonstram-se em maior gravidade, culminando com a ocorrência de exostoses, são indicadas intervenções cirúrgicas, as quais incluem ostectomias e artrodeses. A radioterapia paliativa também tem sido utilizada para pacientes Scottish Fold osteocondrodisplásicos com sintomas avançados da doença (Mathews et al., 1995; Hubler et al., 2004; Fujiwara-Igarashi et al., 2015). No felino atendido tais abordagens não foram consideradas devido ao grau discreto da doença apresentado pelo paciente em questão, no entanto devido ao caráter progressivo da osteocondrodisplasia tais condutas podem vir a ser preconizadas para o animal com o avançar da afecção.

A Federação Internacional de Felinos e outros órgãos não recomendam a reprodução dos Scottish Folds, devido ao comprometimento da qualidade de vida dos animais (Malik, 2001; Gunn-Moore et al., 2008).

Além da osteocondrodisplasia descrita no presente estudo existem relatos de animais Scottish Fold com alterações oculares, auditivas, assim como evidências de predisposição da raça quanto à formação de urólitos de oxalato de cálcio (Mathews et al., 1995; Lekcharoensuk et al., 2000; Inoue et al., 2016).

Este é o primeiro relato de Osteocondrodisplasia em um Scottish Fold no Brasil.

\section{Conclusão}

As deformidades osteoarticulares observadas no felino jovem da raça Scottish Fold com osteocondrodisplasia constituem uma preocupação, em função do comprometimento da qualidade de vida do paciente e ausência de um tratamento curativo para tal afecção, assim o estudo pôde expandir informações a respeito das características inerentes a este padrão racial. 


\section{Referências}

Aydin, D., Altunatmaz, K., Olğun Erdikmen, D., Özer, K., Durmuş, D., \& Avanus, K. (2015). Hereditary osteochondrodysplasia in Scottish Fold cats. Kafkas Üniversitesi Veteriner Fakültesi Dergisi, 21(4), 463-469.

Bennett, D., Zainal Ariffin, S. M., \& Johnston, P. (2012). Osteoarthritis in the cat: 2. How should it be managed and treated? Journal of Feline Medicine and Surgery, 14(1), 76-84. http://dx.doi.org/10.1177/1098612X11432829. PMid:22247327.

Chang, J., Jung, J., Oh, S., Lee, S., Kim, G., Kim, H., Kweon, O., Yoon, J., \& Choi, M. (2007). Osteochondrodysplasia in three Scottish Fold cats. Journal of Veterinary Science, 8(3), 307-309. http://dx.doi.org/10.4142/jvs.2007.8.3.307. PMid:17679781.

Davies, M. (2016). Veterinary clinical nutrition: success stories: an overview. The Proceedings of the Nutrition Society, 75(3), 392-397. http://dx.doi.org/10.1017/S002966511600029X. PMid:27269202.

Frye, C. W., Shmalberg, J. W., \& Wakshlag, J. J. (2016). Obesity, exercise and orthopedic disease. Veterinary Clinics of North America: Small Animal Practice, 46(5), 831-841. http://dx.doi.org/10.1016/j.cvsm.2016.04.006. PMid:27289253.

Fujiwara-Igarashi, A., Igarashi, H., Hasegawa, D., \& Fujita, M. (2015). Efficacy and Complications of Palliative Irradiation in Three Scottish Fold cats with osteochondrodysplasia. Journal of Veterinary Internal Medicine, 29(6), 1643-1647. http://dx.doi.org/10.1111/jvim.13614. PMid:26365740.

Gandolfi, B., Alamri, S., Darby, W. G., Adhikari, B., Lattimer, J. C., Malik, R., Wade, C. M., Lyons, L. A., Cheng, J., Bateman, J. F., McIntyre, P., Lamandé, S. R., \& Haase, B. (2016). A dominant TRPV4 variant underlies osteochondrodysplasia in Scottish fold cats. Osteoarthritis and Cartilage, 24(8), 1441-1450. http://dx.doi. org/10.1016/j.joca.2016.03.019. PMid:27063440.

Graeme, S. A. (2000). Radiographic features of feline joint diseases. The Veterinary Clinics of North America. Small Animal Practice, 30(2), 281-302, vi. http://dx.doi.org/10.1016/S0195-5616(00)50023-0. PMid:10768235.

Gunn-Moore, D., Bessant, C., \& Malik, R. (2008). Breed-related disorders of cats. The Journal of Small Animal Practice, 49(4), 167-168. http://dx.doi.org/10.1111/j.1748-5827.2008.00572.x. PMid:18339086.

Hardie, E. M. (1997). Management of osteoarthritis in cats. The Veterinary Clinics of North America. Small Animal Practice, 27(4), 945-953. http://dx.doi.org/10.1016/S0195-5616(97)50088-X. PMid:9243789.

Hubler, M., Volkert, M., Kaser-Hotz, B., \& Arnold, S. (2004). Palliative irradiation of Scottish fold osteochondrodysplasia. Veterinary Radiology \& Ultrasound, 45(6), 582-585. http://dx.doi.org/10.1111/j.1740-8261.2004.04101.x. PMid:15605854.

Inoue, M., Hasegawa, A., \& Sugiura, K. (2016). Morbidity pattern by age, sex and breed in insured cats in Japan (2008-2013). Journal of Feline Medicine and Surgery, 18(12), 1013-1022. http://dx.doi.org/10.1177/1098612X15616433. PMid:26581469.

Lascelles, B. D. (2010). Feline Degenerative Joint Disease. Veterinary Surgery, 39(1), 2-13. http://dx.doi.org/10.1111/ j.1532-950X.2009.00597.x. PMid:20210938.

Lekcharoensuk, C., Lulich, J. P., Osborne, C. A., Koehler, L. A., Urlich, L. K., Carpenter, K. A., \& Swanson, L. L. (2000). Association between patient-related factors and risk of calcium oxalate and magnesium ammonium phosphate urolithiasis in cats. Journal of the American Veterinary Medical Association, 217(4), 520-525. http:// dx.doi.org/10.2460/javma.2000.217.520. PMid:10953716.

Malik, R. (2001). Genetic diseases of cats. Journal of Feline Medicine and Surgery, 3(2), 109-113. http://dx.doi. org/10.1053/jfms.2001.0121. PMid:11876626.

Malik, R., Allan, G. S., Howlett, C. R., Thompson, D. E., James, G., McWhirter, C., \& Kendall, K. (1999). Osteochondrodysplasia in Scottish Fold cats. Australian Veterinary Journal, 77(2), 85-92. http://dx.doi. org/10.1111/j.1751-0813.1999.tb11672.x. PMid:10078353.

Mathews, K. G., Koblik, P. D., Knoeckel, M. J., Pool, R. R., \& Fyfe, J. C. (1995). Resolution of lameness associated with Scottish fold osteodystrophy following bilateral mastectomies and pantarsal arthrodeses: a case report. Journal of the American Animal Hospital Association, 31(4), 280-288. http://dx.doi.org/10.5326/15473317-31-4280. PMid:7552658.

Partington, B. P., Williams, J. F., Pechman, R. D., \& Beach, R. T. (1996). What is your diagnosis? Scottish Fold osteodystrophy. Journal of the American Veterinary Medical Association, 209(7), 1235-1236. PMid:8837640.

Takanosu, M., Takanosu, T., Suzuki, H., \& Suzuki, K. (2008). Incomplete dominant osteochondrodysplasia in heterozygous Scottish Fold cats. The Journal of Small Animal Practice, 49(4), 197-199. http://dx.doi.org/10.1111/ j.1748-5827.2008.00561.x. PMid:18339089.

Takeuchi, Y., \& Mori, Y. (2009). Behavioral profiles of feline breeds in Japan. The Journal of Veterinary Medical Science, 71(8), 1053-1057. http://dx.doi.org/10.1292/jvms.71.1053. PMid:19721357. 